\title{
Integrative bioinformatics and experimental analysis revealed TEAD as novel prognostic target for hepatocellular carcinoma and its roles in ferroptosis regulation
}

\author{
Xinxin Ren ${ }^{1,}{ }^{*}$, Xiang Wang ${ }^{2,}{ }^{*}$, Yuanliang Yan ${ }^{2}$, Xi Chen $^{2}$, Yuan Cai ${ }^{3}$, Qiuju Liang ${ }^{2}$, Bi Peng ${ }^{3}$, Zhijie \\ $\mathrm{Xu}^{3,4}$, Qingchun $\mathrm{He}^{5,6}$, Fanhua Kang ${ }^{7}$, Jianbo $\mathrm{Li}^{7}$, Wenqin Zhang7, Qianhui Hong7 ${ }^{7}$ Jinwu Peng ${ }^{3,7}$, \\ Muzhang Xiao ${ }^{8}$
}

\author{
${ }^{1}$ Center for Molecular Medicine, Xiangya Hospital, Key Laboratory of Molecular Radiation Oncology of Hunan \\ Province, Central South University, Changsha 410008, Hunan, China \\ ${ }^{2}$ Department of Pharmacy, Xiangya Hospital, Central South University, Changsha 410008, Hunan, China \\ ${ }^{3}$ Department of Pathology, Xiangya Hospital, Central South University, Changsha 410008, Hunan, China \\ ${ }^{4}$ National Clinical Research Center for Geriatric Disorders, Xiangya Hospital, Central South University, Changsha \\ 410008, Hunan, China \\ ${ }^{5}$ Department of Emergency, Xiangya Hospital, Central South University, Changsha 410008, Hunan, China \\ ${ }^{6}$ Department of Emergency, Xiangya Changde Hospital, Changde 415000, Hunan, China \\ ${ }^{7}$ Department of Pathology, Xiangya Changde Hospital, Changde 415000, Hunan, China \\ ${ }^{8}$ Department of Burn and Plastic Surgery, Xiangya Hospital, Central South University, Changsha 410008, Hunan, \\ China \\ *Equal contribution and share first authorship
}

Correspondence to: Qingchun He, Jinwu Peng; email: 404113@csu.edu.cn, jinwupeng@csu.edu.cn Keywords: TEAD family, hippo pathway, hepatocellular carcinoma, ferroptosis, immune infiltration Received: November 22, 2021 Accepted: January 17, 2022

Published: January 25, 2022

Copyright: (c) 2022 Ren et al. This is an open access article distributed under the terms of the Creative Commons Attribution License (CC BY 3.0), which permits unrestricted use, distribution, and reproduction in any medium, provided the original author and source are credited.

\section{ABSTRACT}

Objective: Transcriptional enhanced associate domain (TEAD) family consists of four members TEAD1/2/3/4 that regulate cell growth, stem cell functions and organ development. As the downstream of Hippo signaling pathway, TEAD family is involved in the progression of several cancers. However, the precise biology functions of TEAD family in hepatocellular carcinoma (HCC) have not been reported yet.

Methods: We apply bioinformatics analysis based on databases including UALCAN, Oncomine, GEPIA, KaplanMeier plotter, WebGestalt, cBioPortal, TIMER2.0, and in vitro experimental evidence to identify the exact roles of TEAD family in HCC.

Results: The results indicated that TEAD2/4 were significantly upregulated in HCC compared with normal tissues. Downregulated of TEAD2 could promote the death of HCC cells through inducing ferroptosis by iron accumulation and subsequent oxidative damage. According to the Kaplan-Meier plotter database, we found that the high expression of TEAD2 was significantly associated with poor disease-specific survival, overall survival, progression-free survival and relapse-free survival. In aspect of cancer immunity, Tumor Immune Estimation Resource algorithm showed that the expression of TEAD family members was obviously related to multiple of infiltrating immune cells including macrophages, neutrophils, dendritic cells, B cells, CD8+ T cells and CD4+ $T$ cells. Finally, we conducted the functional enrichment analysis including protein-protein interaction 


\section{network, gene ontology enrichment analysis and Kyoto Encyclopedia of Genes and Genomes pathway based on the TEAD family-associated coexpression genes. \\ Conclusion: The study provided deep insight information of TEAD family in the diagnostic and prognostic evaluation of HCC patients.}

\section{INTRODUCTION}

The occurrence of liver cancer is rising gradually [1]. Research estimates that there will be over 1 million individuals that develop liver cancer per year by 2025 [2]. Hepatocellular carcinoma (HCC) occupies about $90 \%$ of liver cancer patients [3]. At present, we apply chemoradiotherapy and surgery to deal with the early stage of HCC in clinical and obviously improve the patients' condition [4]. However, the majority of patients are diagnosed in advanced stages [5]. In addition, the molecular pathogenesis of $\mathrm{HCC}$ varies on the basis of the different etiologies and genotoxic insults [6]. Then, searching for novel molecular targets are crucial for the diagnosis and prognosis of HCC.

Transcriptional enhanced associate domain (TEAD) family take vital effects in the organism [7]. The TEAD family contains four members (TEAD1, TEAD2, TEAD3 and TEAD4), which each have tissue-specific roles, including trophectoderm, cardiogenesis, lineage determination and neural development [8]. TEAD family is regarded to be primarily combine to the DNA and it is also the downstream nuclear effectors of the Hippo pathway [9]. As we all know, Hippo pathway plays essential roles in cell proliferation through its downstream target genes, which has a crucial impact on tumorigenesis $[10,11]$. Therefore, as reported, TEAD family was strongly related to the development of cancer and had an influence on several cancers including prostate cancer, ovarian cancer and lung cancer [12-14]. Moreover, YAP/TAZ could promote HCC cells to overcome sorafenib-induced ferroptosis in a TEAD-dependent manner, which suggested that there was a relationship between TEAD family and HCC [15]. Therefore, the roles of TEAD family in HCC are urgent to be illustrated.

Ferroptosis is always triggered by lipid oxidation [16]. Ferroptosis is involved in the development of several tumors, including HCC $[17,18]$. Hippo signaling pathway is mediated by YAP) and TAZ, which interacted with TEAD proteins for gene expression regulation [19]. At present, YAP/TAZ has been found to be as the novel determinants of ferroptosis. In addition, YAP promotes ferroptosis via the E3 Ligase SKP2 in renal and ovarian cancer cells [20, 21]. However, the relationship between TEAD and ferroptosis has not been illustrated.
In the study, using bioinformatics databases and experimental verification, we discussed the effects of TEAD family in HCC (Supplementary Table 1). Furthermore, the potential as diagnostic biomarkers and prognostic targets were evaluated. Moreover, downregulated the expression of TEAD2 could promote ferroptosis in HCC cell lines. Through the comprehensive analysis, TEAD family members, especially TEAD2, contributed to helping clinicians proved better medical services for HCC patients.

\section{MATERIALS AND METHODS}

\section{Cell culture}

The normal hepatocyte HHL-5 and HCC parental cell lines PVTT, QGY, Huh7, MHCC97H, 7404 and 7721 were kindly provided by Professor Yuezhen Deng (Central South University, China). QGY cells were cultured by using 1640 medium (Gibco, Invitrogen), while other cells were cultured in DMEM, which contained 10\% fetal bovine serum (FBS, Gibco) and 1\% penicillin and streptomycin, incubation under the condition at $37^{\circ} \mathrm{C}$ with $5 \% \mathrm{CO}_{2}$.

\section{Antibodies and chemicals}

Antibodies and chemicals were employed in the study: TEAD2 (21159-1-AP, Proteintech), Actin (66009-1-Ig, Proteintech), erastin (B1524, APExBIO), RSL3 (B6095, APExBIO), Ferrostatin-1 (A4371, APExBIO), Z-VADFMK (A1902, APExBIO), Necrostatin-1 (A4213, APExBIO).

\section{Transfections}

Lipofectamine 3000 was employed to perform siRNA transfection based on the manufacture's protocol. The sense sequences of siTEAD2 were settled bellow: siTEAD2-1-GAGTGAGCAGCCAGTATGA, siTEAD2-2-GGTTGCAGCTGGTAGAGTT.

\section{qPCR}

The PrimeScriptTM RT reagent kit (6210, Takara) was applied to converted the total RNA to cDNA. The qPCR assay was conducted with SYBR green kit (1725121, Bio-Rad), and 18S rRNA was employed for an internal control. We listed the primer sequences of TEAD family in Table 1 and confirmed the relative expression levels. 
Table 1. The primer sequences of TEAD family involved in RT-PCR.

\begin{tabular}{lcc}
\hline Primers & Forward sequences & Reverse sequences \\
\hline TEAD1 & ATGGAAAGGATGAGTGACTCTGC & TCCCACATGGTGGATAGATAGC \\
TEAD2 & CTTCGTGGAACCGCCAGAT & GGAGGCCACCCTTTTTCTCA \\
TEAD3 & TCATCCTGTCAGACGAGGG & TCTTCCGAGCTAGAACCTGTATG \\
TEAD4 & GAACGGGGACCCTCCAATG & GCGAGCATACTCTGTCTCAAC \\
\hline
\end{tabular}

Gene expression profiling interactive analysis (GEPIA)

GEPIA is a database used to provide numerous GTEx and TCGA data [22]. We explored the level of TEAD family member in HCC tissues compared with normal tissues.

\section{UALCAN}

UALCAN is a public web tool employed for exploring gene expression and survival information [23]. We evaluated TEAD family expression in HCC patients.

\section{GE-mini}

GE-mini database is generally used for the basic simple analysis of gene mutations, co-expressed/co-mutated genes [24]. We discussed the level of TEAD in HCC.

\section{Oncomine 3.0}

Oncomine database integrates lots of data for the bioinformatic analysis [25]. From the database, the level of TEAD family in HCC patients was tested.

\section{Western blot}

The protein concentration was tested and the separation operation is then performed. The membranes were incubated with the corresponding primary antibodies at $4^{\circ} \mathrm{C}$ overnight. Next day, the secondary antibodies were kept together with the membranes for $1-2 \mathrm{~h}$ and chemiluminescence reagent was applied to detect the immunoreaction signals.

\section{Cell viability assay}

Cells were cultured with a density of 900 cells/well in a 96-well plate (). Then, the $10 \%$ CCK8 was added to the medium. The absorbance was calculated at $450 \mathrm{~nm}$.

\section{Reactive oxygen species (ROS) assay}

ROS levels were tested applying DCFDA/H2DCFDA Kit (ab113851, Abcam). We used the DCFDA Solution to stain cells away from light. Next, we employed the microscopy to detect fluorescence intensity.

\section{Iron assay}

We used the iron assay kit (ab83366, Abcam) to test the $\mathrm{Fe}^{2+}$ level. Generally, $1 \times 10^{6}$ cells were mixed together with $100 \mu \mathrm{l}$ iron assay buffer. Then, the iron reducer was added and incubated for $30 \mathrm{~min}$. Subsequently, the iron probe was added, then, tested at $593 \mathrm{~nm}$.

\section{Malondialdehyde (MDA) assay}

A Lipid Peroxidation Kit (MAK085, Sigma) was used to quantify the MDA concentration. The thiobarbituric acid (TBA) was applied to test the amounts of MDA at $531 \mathrm{~nm}$.

\section{Kaplan-Meier plotter}

Kaplan-Meier plotter is conducted based on gene chip and RNA-seq data obtained from public databases [26]. We assessed the association between TEAD family members and the prognosis of HCC patients.

\section{cBioPortal}

cBioPortal provides a web-based database for cancer related data analysis [27]. We employed the web for understanding the biology functions in $\mathrm{HCC}$ tissues.

\section{Protein-protein interaction (PPI)}

The STRING database was planned for conducting the PPInetworks [28]. Here, we established the PPI network of TEAD family using STRING and Cytoscape.

\section{WebGestalt}

WebGestalt is an online website concentrating on enrichment analysis and supporting multiple enrichment analysis algorithms [29]. We obtained the Gene ontology (GO) enrichment analysis and Kyoto Encyclopedia of Genes and Genomes (KEGG) pathway through the web tool [30].

\section{TIMER2.0}

The database is mainly performed to analyzing the relationship between immune infiltration and gene expression [31]. 


\section{Statistical analyses}

All the results were independently repeated at least three times and represent as mean $\pm \mathrm{SD}$. Student's $t$-test was employed to for proper analysis. $P<0.05$ was regarded to be statistical significance.

\section{RESULTS}

\section{TEAD family was abnormal expressed in HCC}

Above all, we used the GEPIA database to explore the mRNA expression of TEAD family in HCC.
TEAD1/2/4 were upregulated in HCC, while TEAD3 was downregulated (Figure 1A). In the Wurmbach Liver derived from Oncomine database, TEAD1/2/3/4 expression was increased in HCC (Figure 1B). Then, we also evaluated the expression of TEAD family in UNCLAN database, revealing that TEAD1/2/3/4 were all obviously higher in HCC to normal liver (Figure 1C). In addition, TEAD2/3/4 were evidently upregulated in HCC tissues in contrast to normal liver, however, TEAD1 was downregulated (Figure 1D). Finally, we conducted the in vitro experiment and RTPCR presented that the mRNA expression of TEAD1/2/3/4 were all upregulated in Huh7 and
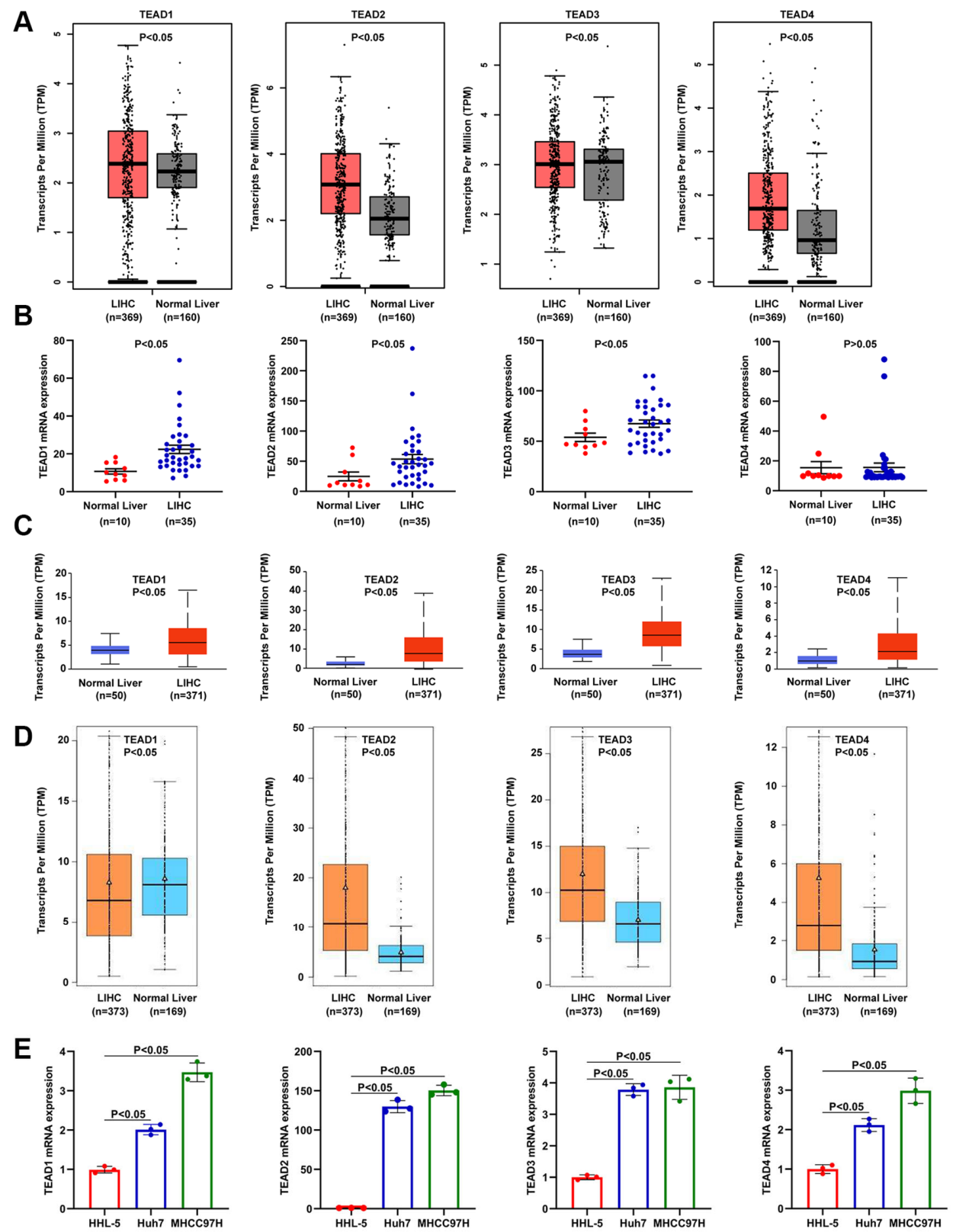

Figure 1. The mRNA level of TEAD family in HCC. (A-D) The mRNA level of TEAD members in HCC acquired from GEPIA, Oncomine, UNCLAN, and GE-mini databases. The HCC tissues and normal tissues were showed by T and N, respectively. (E) Levels of TEAD family members expressed in normal liver cell line HHL-5 and HCC cell lines Huh7 and MHCC97H experimented by RT-PCR. 
MHCC97H cells (Figure 1E). These data illustrated that TEAD family might have roles on HCC patients.

\section{The prognostic of the TEAD family in HCC patients}

Next, we discussed the influence of TEAD family on HCC patients' survival. Based on the database, the mechanism of TEAD family expression in HCC patients' prognosis was uncovered. As for diseasespecific survival (DSS) and overall survival (OS), TEAD2/4 higher expression marked with a shorter DSS time, as well as OS time (Figure 2A, 2B). In terms of progression-free survival (PFS), the upregulated of TEAD2/3 had a powerful association with poor PFS (Figure 2C). Moreover, patients with higher expression of TEAD2/3 presented short relapse-free survival
(RFS), while the lower expression of TEAD4 was related to poor RFS (Figure 2D). These results told us that TEAD family, especially TEAD2, had the potential to forecast the prognosis of HCC patients.

\section{Functional analysis of TEAD family associated coexpressed genes}

In order to survey the exact roles of TEAD family in HCC patients, we created a comprehensive biological function analysis. From the cBioPortal database, 19272 genes were downloaded. Then, we chose the top 200 coexpressed molecules most related to TEAD family to create a PPI network by using STRING and Cytoscape software (Supplementary Table 2). Results revealed that EP300 and BPTF were mainly correlated with the
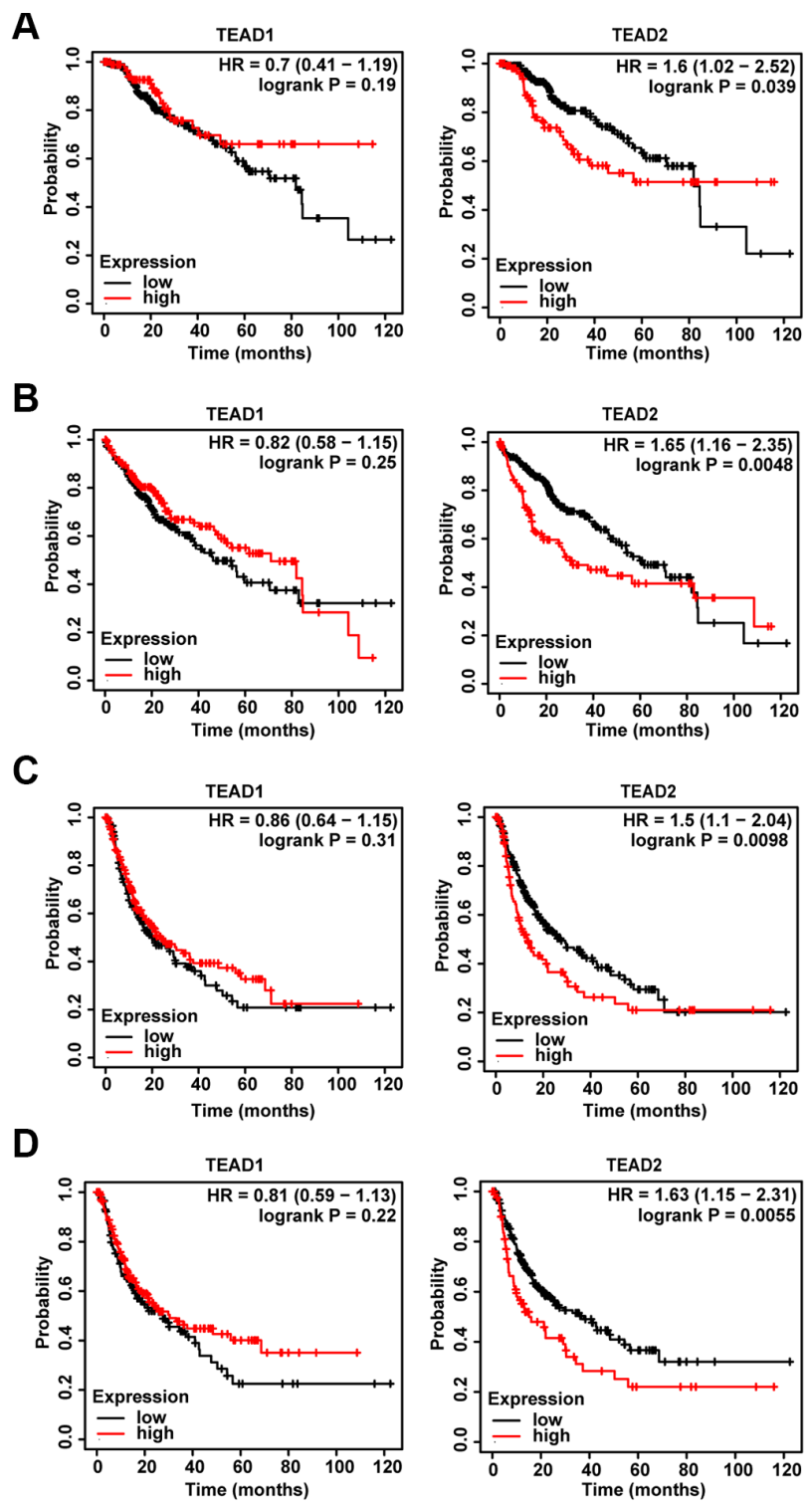
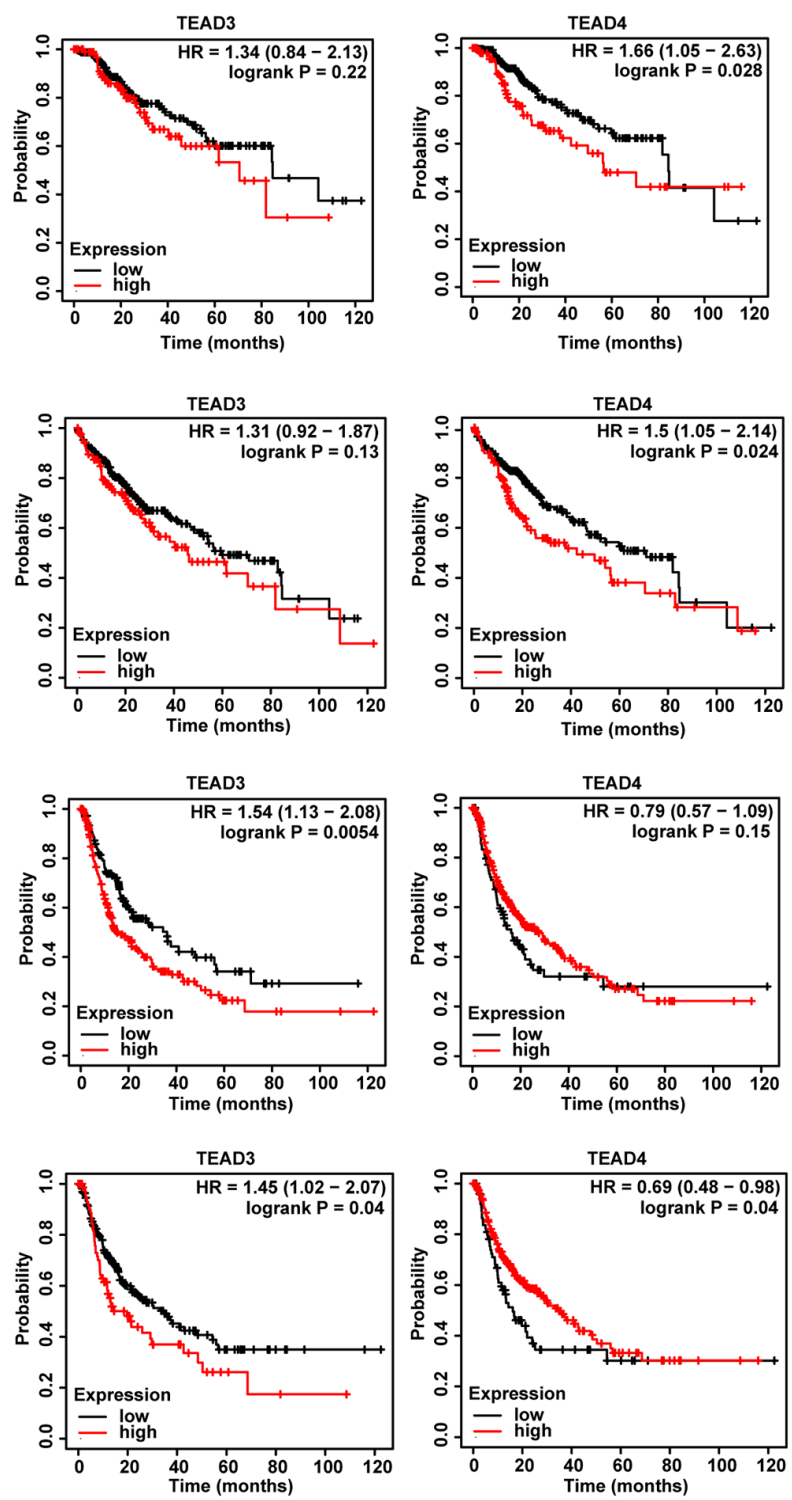

Figure 2. The effect of TEAD family on the survival of HCC patients. (A-D) The association of TEAD family with DSS, OS, PFS and RFS in HCC patients obtained from Kaplan-Meier plotter database. 
TEAD family regulation (Figure 3A). Next, we employed the WebGestalt web tool to completed the GO and KEGG analysis. In terms of biological processes, the metabolism was mainly for TEAD family participated (Figure 3B). In addition, TEAD family was primarily enriched in membrane (Figure 3B). Finally, TEAD family was obviously related to nucleobasecontaining small molecule interconversion and nucleobase metabolic process (Figure 3C).

\section{TEAD family influence on the immune cell infiltration in HCC}

To explore the connection between the two aspects, we acquired the information from TIMER database. Results showed that TEAD1 was positively associated with B cells, CD8 $+\mathrm{T}$ cells, CD4+ $\mathrm{T}$ cells, macrophages, neutrophils and dendritic cells (Figure 4A). Moreover, the expression of TEAD2/3/4 was positively related to the immune cells (Figure 4B-4D). Meanwhile, we further found that there was an obviously association between the clinical outcome and immune cells in HCC patients. (Table 2).

\section{TEAD2 acts as a repressor of ferroptosis}

From the above studies, we concluded that TEAD2 was upregulated in HCC. Then, we explored the protein level of TEAD2and TEAD2 was higher in PVTT, QGY, Huh7, MHCC97H, 7404 and 7721 cells, compared to HHL-5 cell (Figure 5A). Studies had shown that ferroptosis played an essential role in the biology of $\mathrm{HCC}$ and ferroptosis-related genes were contributed to predicting the prognosis in $\mathrm{HCC}$ [32]. In addition, suppressing YAP could promote HCC cells sensitive to ferroptosis [33]. Thus, we want to discuss the roles of TEAD2 in ferroptosis. Then, we knocked down TEAD2 in Huh7 and MHCC97H (Figure 5B, 5C). Next, we used the CCK-8 cell viability assay for cell death analysis after erastin and RSL3, inducers of ferroptosis, treatment in different groups. Results shown that knocked down TEAD2 could obviously promote the death of Huh7 and MHCC97H, whileferrostatin-1 could reverse the phenomenon (Figure 5D). However, ZVADFMK and necrostatin-1 could not effect on cell death induced by erastin and RSL3. This demonstrated that TEAD2 could inhibit the cell death through suppressing the ferroptosis in HCC cells.

As we all know, $\mathrm{Fe}^{2+}$ and lipid peroxidation were participated in ferroptosis [34]. In Figure 5E, inhibited the expression of TEAD2 increased the intracellular $\mathrm{Fe}^{2+}$ accumulation in both Huh7 and MHCC97H cells after erastin and RSL3 treatment, this phenomenon could be reversed by ferrostatin-1. As MDA and ROS were the crucial products of lipid peroxidation, we
A

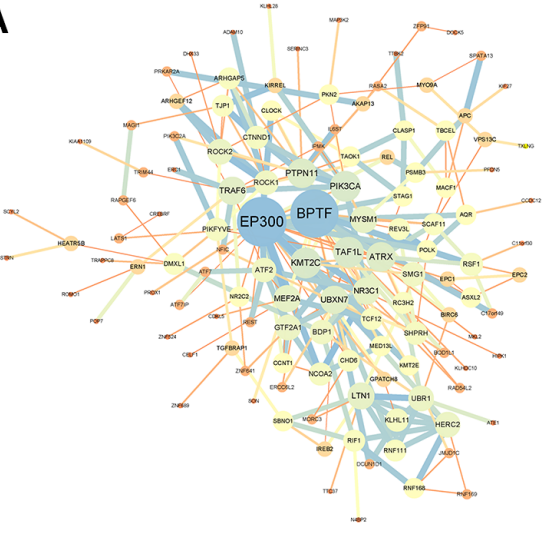

C

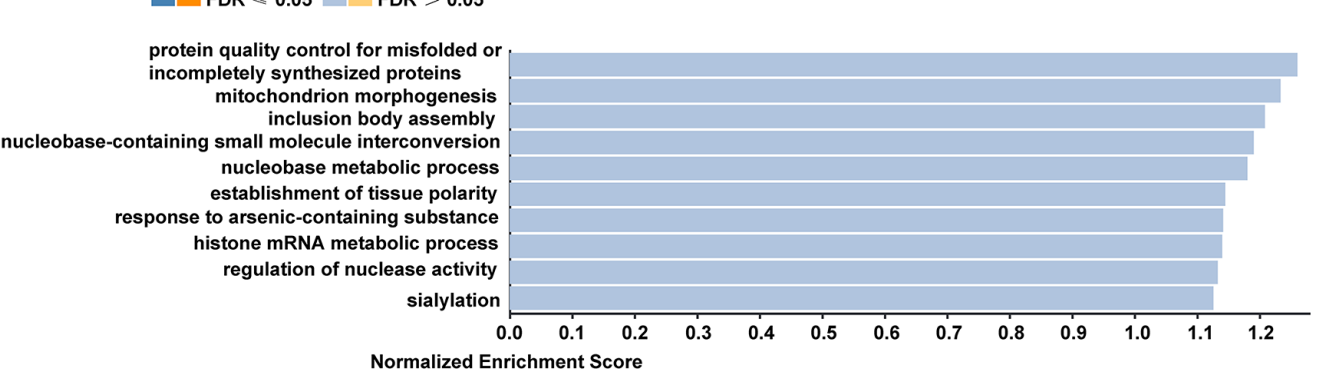

B
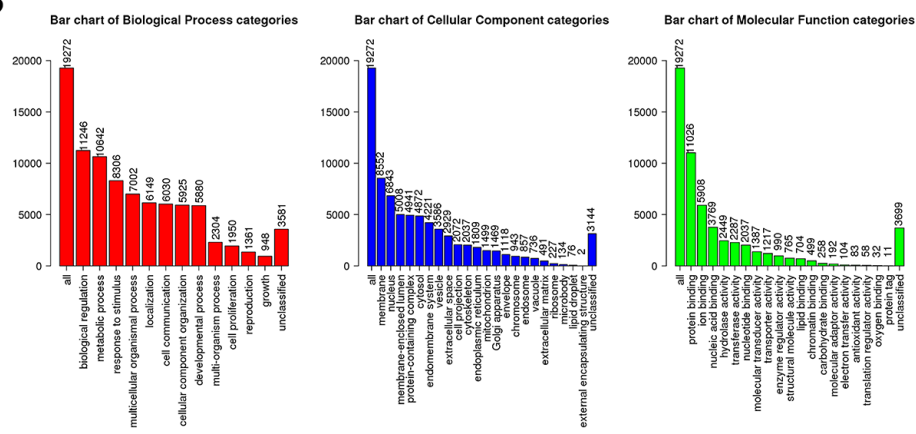

Figure 3. The biological functions of TEAD family in HCC samples. (A) The PPI network based on TEAD family-associated coexpression genes drew by STRING and Cytoscape. (B-C) GO and KEGG analyzed by WebGestalt. 
Table 2. The cox proportional hazard model of the TEAD family and six tumor-infiltrating immune cells in HCC.

\begin{tabular}{|c|c|c|c|c|c|c|}
\hline & coef & HR & 95\% CI_l & 95\% CI_u & p.value & sig \\
\hline B_cell & -8.977 & 0 & 0 & 0.161 & 0.014 & $*$ \\
\hline CD8_Tcell & -6.555 & 0.001 & 0 & 0.16 & 0.006 & ** \\
\hline CD4_Tcell & -5.332 & 0.005 & 0 & 3.166 & 0.107 & \\
\hline Macrophage & 7.24 & 1393.616 & 12.308 & 157797.7 & 0.003 & $* *$ \\
\hline Neutrophil & 4.067 & 58.397 & 0.001 & 4825308 & 0.481 & \\
\hline Dendritic & 6.745 & 850.031 & 24.212 & 29842.46 & 0 & $* * *$ \\
\hline TEAD1 & -0.219 & 0.803 & 0.64 & 1.008 & 0.059 & \\
\hline TEAD2 & 0.125 & 1.134 & 0.954 & 1.347 & 0.154 & \\
\hline TEAD3 & -0.033 & 0.967 & 0.725 & 1.291 & 0.822 & \\
\hline TEAD4 & 0.11 & 1.117 & 0.91 & 1.37 & 0.29 & \\
\hline
\end{tabular}

explored whether the indicator could be impacted by TEAD2. Results displayed TEAD2-deficient elevated the MDA levels in Huh7 and MHCC97H cells and the process could be reversed by ferrostatin-1 (Figure 5F, Figure 6A-6H). These results showed that TEAD2 overexpression could regulate $\mathrm{Fe}^{2+}$ accumulation and lipid peroxidation in HCC cells.

\section{DISCUSSION}

In the study, we revealed the level and prognosis of TEAD family in HCC through bioinformatics analysis as well as experimental verification. TEAD family was abnormal expressed in HCC. In addition, the higher expression of TEAD2/4 was always had shorter DSS

\section{A}
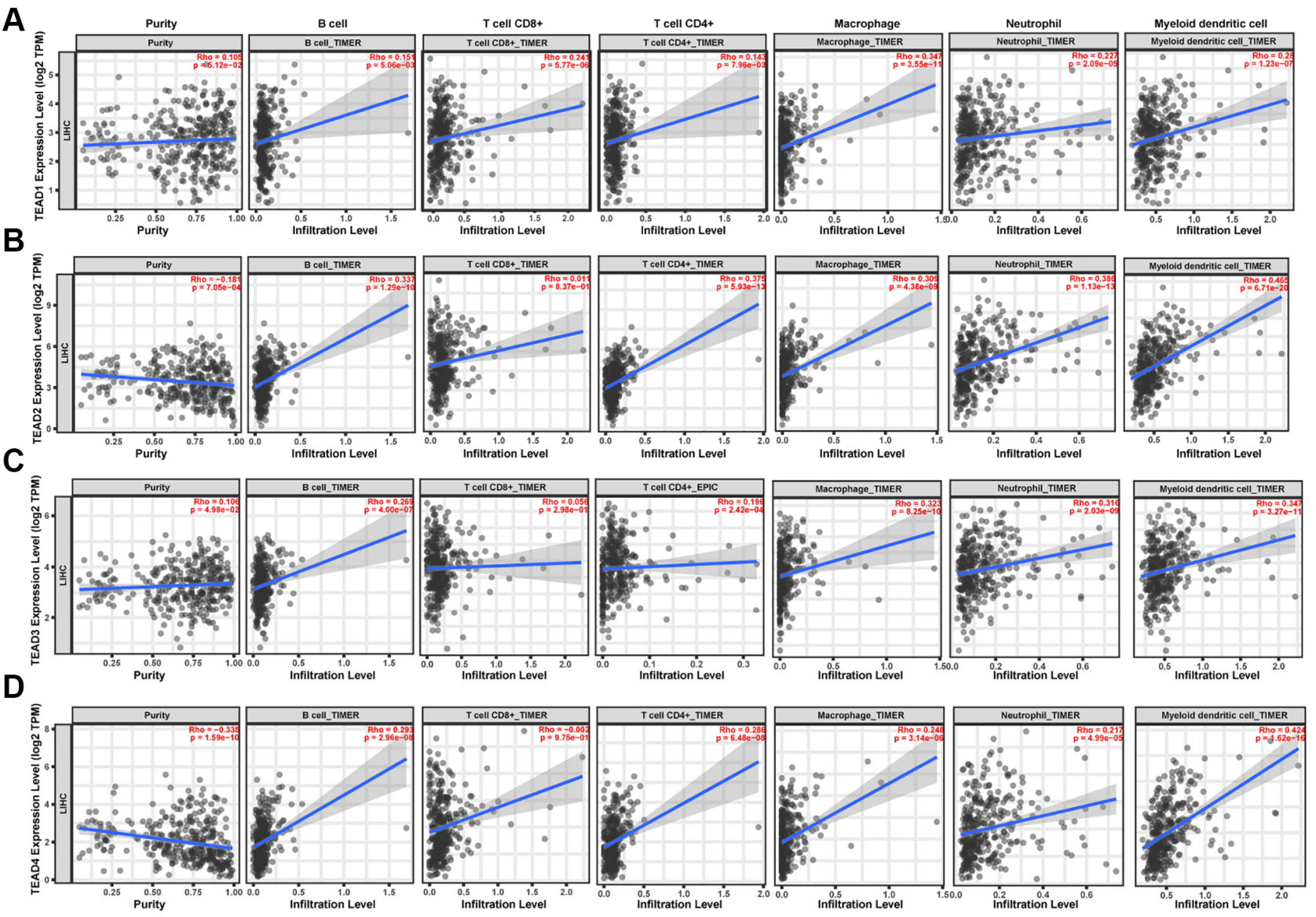

Figure 4. The relationship between the expression of TEAD family and immune cell infiltration in HCC patients. (A-D) The influence of TEAD1/2/3/4 on the immune cell infiltration. 
and OS. Patients with evaluated TEAD2/3 levels had shorter PFS and RFS, while patients with decreased TEAD4 level was connected to poor RFS. TEAD family could influence on the survival of HCC patients. Interestingly, TEAD family could influence the immune cells.

A

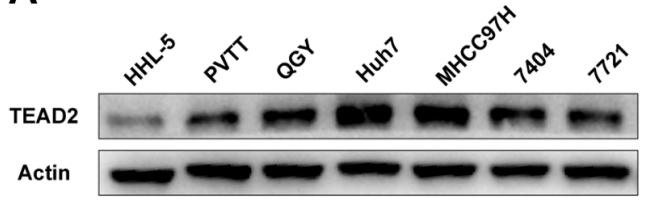

B

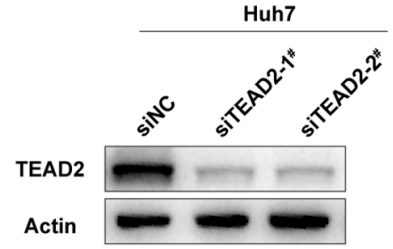

D

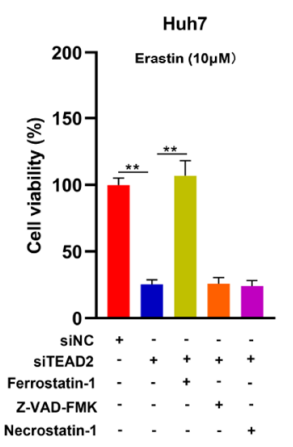

E

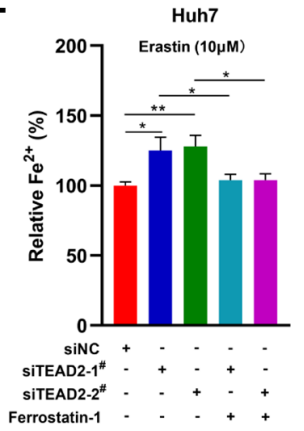

F

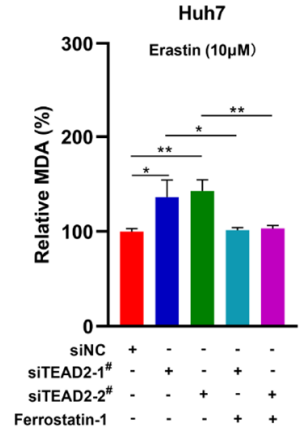

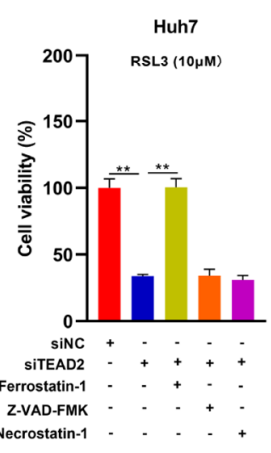
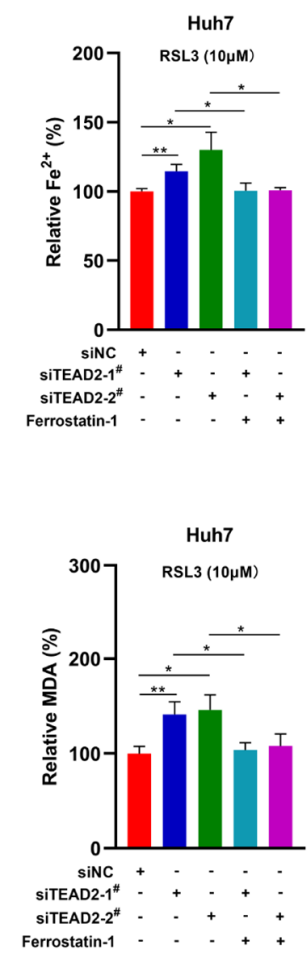

Ferroptosis is different from apoptosis and necrosis [35]. Recently, reports have shown that ferroptosis has a strongly relationship with cancer, including HCC [33]. A series of studies have demonstrated that inducing ferroptosis could promote HCC cells death and inhibit the proliferation of HCC [36]. Moreover, we also find
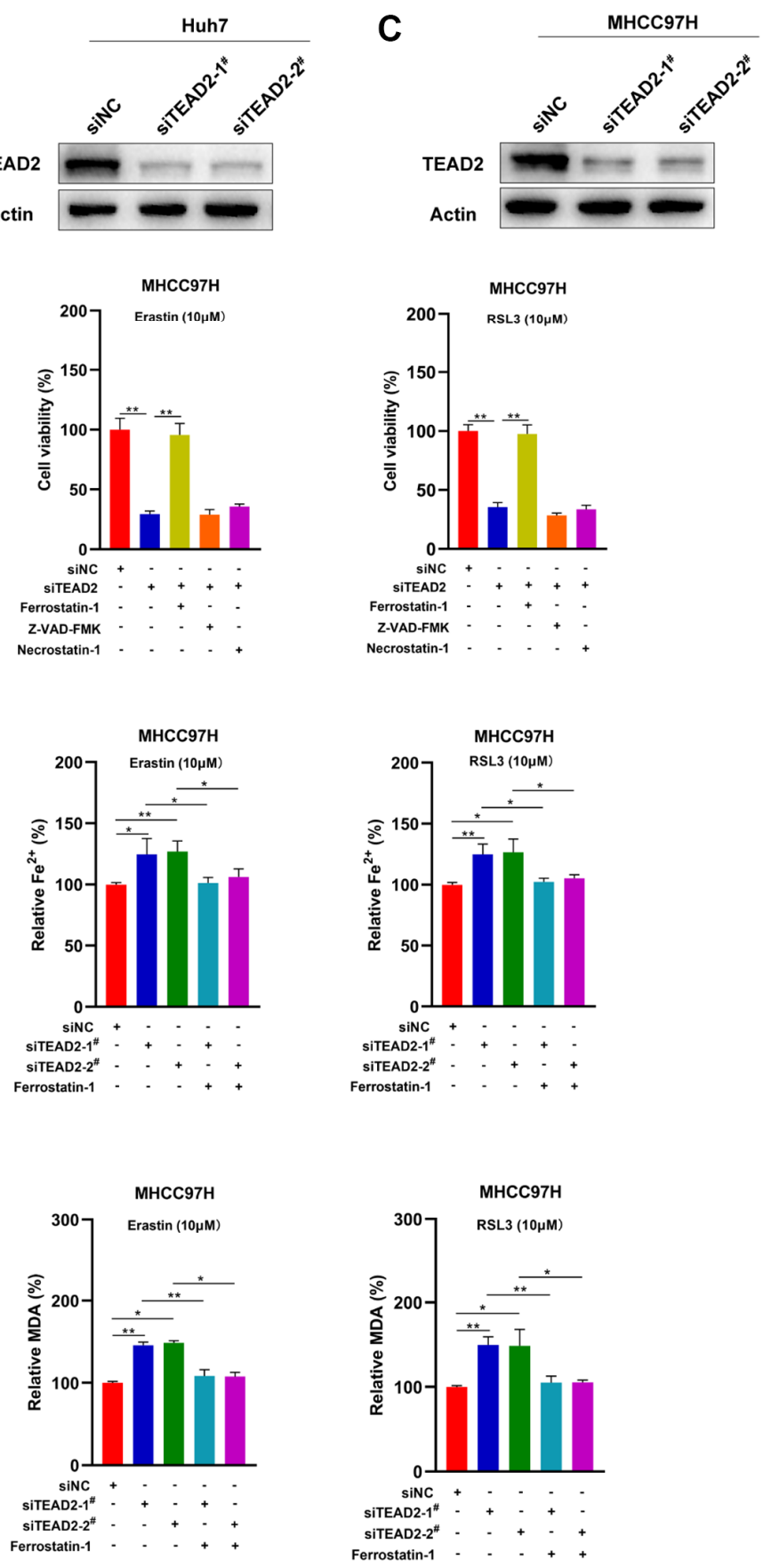

Figure 5. TEAD2 negatively regulates ferroptosis in HCC. (A) The protein levels of TEAD2 in normal liver cell line HHL-5 and HCC cell lines PVTT, QGY, Huh7, MHCC97H, 7404 and 7721. (B-C) Knockout of TEAD2 in Huh7 and MHCC97H cells was confirmed by Western blot. (D) Knocked down of TEAD2 (siTEAD2-1) promoted the cell death induced by erastin and RSL3 in Huh7 and MHCC97H cells. The cells were treated with erastin $(10 \mu \mathrm{M})$ or RSL3 $(10 \mu \mathrm{M})$ with or without ferrostatin-1 $(5 \mu \mathrm{M})$, ZVAD-FMK $(10 \mu \mathrm{M})$ and necrostatin-1 $(5 \mu \mathrm{M})$ for $24 \mathrm{~h}$. They were tested by a CCK-8 kit. (E-F) The expression levels of TEAD2 impacted on the $\mathrm{Fe}^{2+}$ and MDA accumulation in erastin or RSL3treated Huh7 and MHCC97H cells. The cells were treated with erastin $(10 \mu \mathrm{M})$ or RSL3 $(10 \mu \mathrm{M})$ for $24 \mathrm{~h}$. Subsequently, the intracellular Fe ${ }^{2+}$ and MDA were assayed. Data was represented with mean \pm SD $(n=3) .{ }^{*} p<0.05 ;{ }^{* *} p<0.01$. 
that knock out TEAD2 could promote ferroptosis and accelerate HCC cells death. However, ferroptosis enable to promote the HCC incidence by altering tumor microenvironment [37]. These studies suggested that ferroptosis had a dual role in HCC. Nonetheless, the specific mechanisms of ferroptosis in HCC had not been clarified, which needed further research. In addition, we found that TEAD family was influenced on the immune cells. Reports have demonstrated that TEAD could coordinate several signal pathways such as Hippo, EGFR, Wnt and TGF $\beta$ [38]. The abnormal expression of TEAD could modulate several cancer-associated genes, including MYC, KRAS, NF2, BRAF and LKB1, which had crucial roles in the regulation of tumor

\section{A}

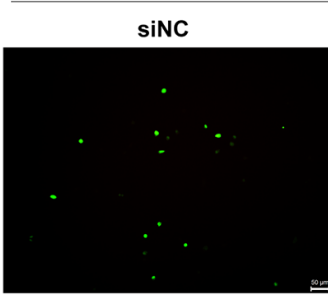

B

siNC

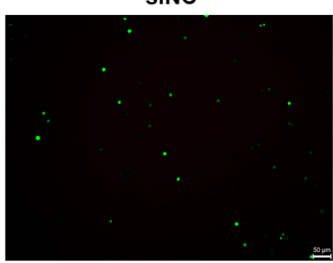

C

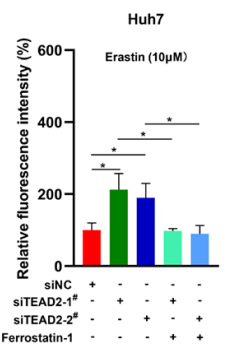

D

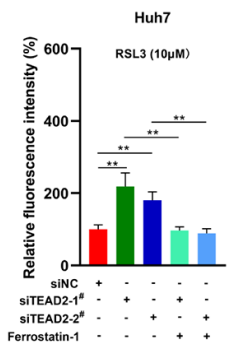

siTEAD2-1 ${ }^{\#}$

Huh7 (Erastin=10 $\mu \mathrm{M})$

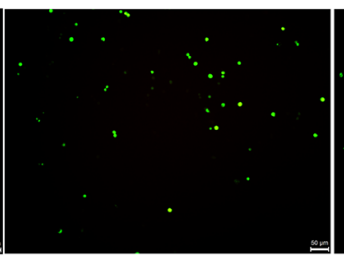

SITEAD2-2

siTEAD2-1"+Ferrostain-1

siTEAD2-2 ${ }^{\sharp}+$ Ferrostain-1
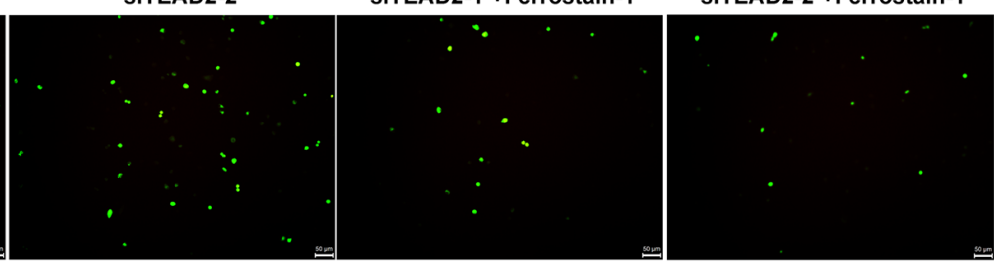

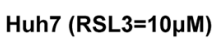

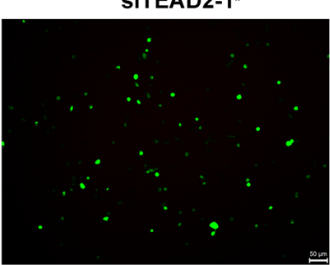

siTEAD2-2 ${ }^{\#}$

siTEAD2-1"+Ferrostain-1

siTEAD2-2 ${ }^{\#+F e r r o s t a i n-1}$
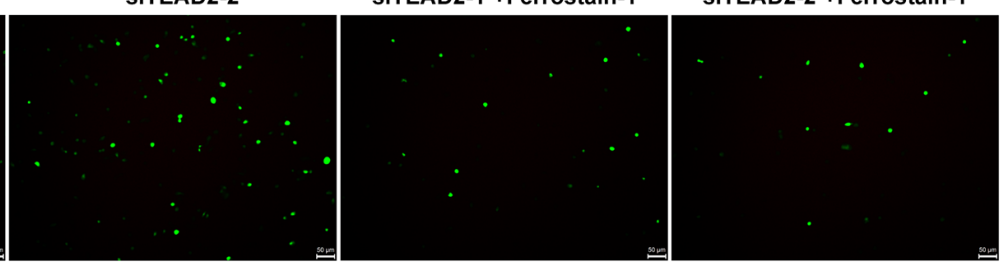

G

H
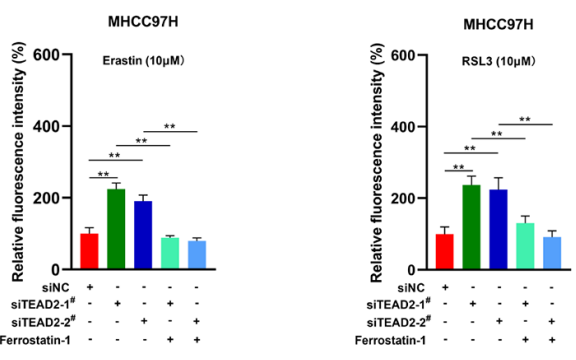

E

MHCC97H (Erastin=10 $\mu \mathrm{M})$
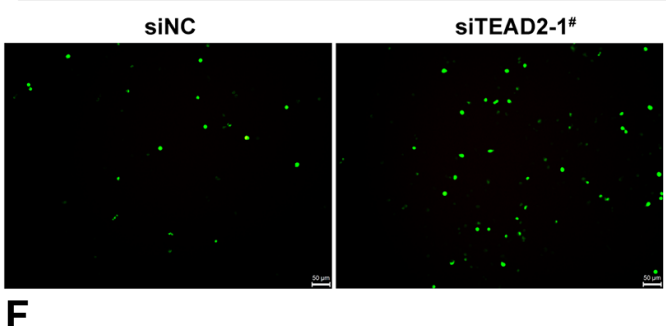

F

siNC

siTEAD2-1"
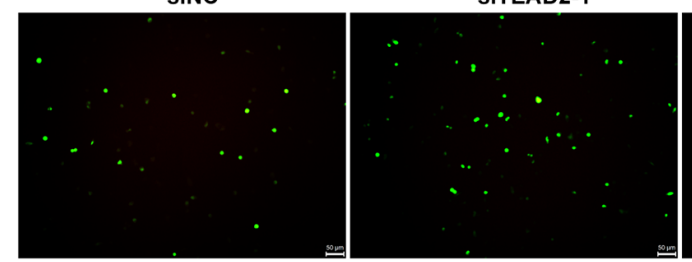

SITEAD2-2

siTEAD2-1 ${ }^{\#+F e r r o s t a i n-1}$

siTEAD2-2 ${ }^{\#}+$ Ferrostain-1
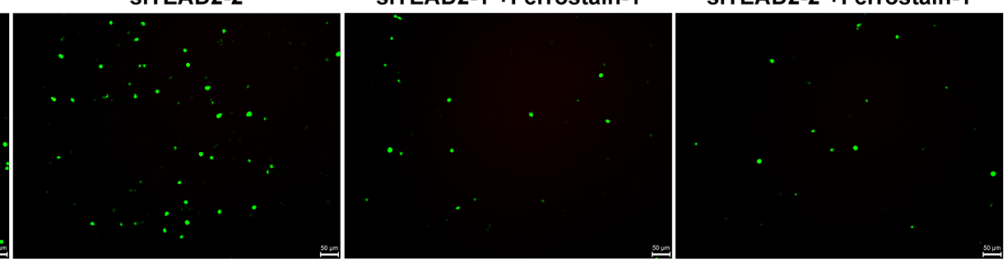

MHCC97H (RSL3=10 $\mu \mathrm{M})$

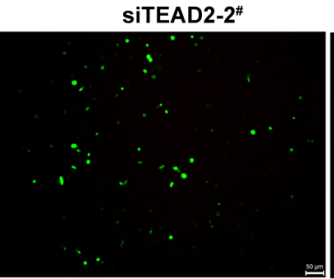

siTEAD2-1"+Ferrostain-1

siTEAD2-2 ${ }^{\#+F e r r o s t a i n-1}$

Figure 6. TEAD2 regulates the ROS levels of HCC. (A-H) The expression levels of TEAD2 impacted on the intracellular ROS levels following the treatment of erastin $(10 \mu \mathrm{M})$ or RSL3 $(10 \mu \mathrm{M})$ on Huh7 and MHCC97H cells. The cells were treated with erastin $(10 \mu \mathrm{M})$ or RSL3 $(10 \mu \mathrm{M})$ for $24 \mathrm{~h}$. Subsequently, the intracellular ROS were assayed. Data was shown with mean \pm SD $(n=3) .{ }^{* *} p<0.01$. 
immunity [39]. These results showed that TEAD might affect the immunological response in tumorigenesis, which deserved to be further study.

The Hippo pathway could integrate a lot of signals to directly or indirectly regulate multiple cancer hallmarks, including proliferation, invasion and metastasis, survival, angiogenesis, evading growth suppressors, as well as inflammation and immunosuppression [40, 41]. For example, the Hippo pathway could regulate the proliferation through PTEN/AKT/mTOR-mediated autophagy in HCC [42]. At present, some studies suggested that the Hippo pathway might have relationship with ferroptosis in cancer [43]. For example, Hippo-YAP/TAZ pathway could increase the sensitivity to erastin-induced ferroptosis in RCC [20]. YAP might control ferroptosis through elevated YAP protein expression in cancer [44]. Moreover, endogenous glutamate enable to enhance ferroptosis sensitivity based on the ADCY10dependent YAP suppression in HCC [45]. In addition, YAP always binds to TEAD to promote a series of gene transcription profiles, which are involved in cell proliferation and differentiation [46]. In the study, we concluded that TEAD family could affect the development of HCC through regulating ferroptosis. These findings illustrated that the Hippo pathway could control the progression of cancer by regulating ferroptosis.

In summary, through the bioinformatics and experimental methods, we revealed the TEAD family functions on HCC. We found that the expression of TEAD2 was evidently related to OS, DSS, PFS and RFS in HCC patients. Excitedly, TEAD2 could influence the occurrence of ferroptosis. Furthermore, TEAD2 expression enabled affect immune infiltration in HCC. These results in the study showed novel biomarker for therapy and survival of HCC patients.

\section{AUTHOR CONTRIBUTIONS}

J Peng, Q He and Z Xu contributed to the conception and design of the study. $X$ Ren and $X$ Wang performed the experiments. X Chen, Y Cai, Q Liang, and B Peng collated the data. $\mathrm{X}$ Ren, $\mathrm{X}$ Wang and $\mathrm{Y}$ Yan contributed to the writing, review, and revision of the manuscript. F Kang, J Li, W Zhang, Q Hong and M Xiao performed the administrative, technical and material support. All authors approved final version of manuscript.

\section{CONFLICTS OF INTEREST}

The authors declare no conflicts of interest related to this study.

\section{FUNDING}

This study is supported by grants from the Science and Technology Innovation Program of Hunan Province (2021RC3029), the China Postdoctoral Science Foundation (2021T140754, 2020M672521), the National Natural Science Foundation of China (81803035), the Natural Science Foundation of Hunan Province (2020JJ5934), and the Postdoctoral Science Foundation of Central South University (248485).

\section{REFERENCES}

1. Zhang J, Huang H, Bian J, Sang X, Xu Y, Lu X, Zhao H. Safety, feasibility, and efficacy of associating liver partition and portal vein ligation for staged hepatectomy in treating hepatocellular carcinoma: a systematic review. Ann Transl Med. 2020; 8:1246.

https://doi.org/10.21037/atm-20-2214

PMID:33178778

2. Li J, Zhu Y. Recent Advances in Liver Cancer Stem Cells: Non-coding RNAs, Oncogenes and Oncoproteins. Front Cell Dev Biol. 2020; 8:548335. https://doi.org/10.3389/fcell.2020.548335 PMID:33117795

3. Ali OM, El Amin HA, Sharkawy YL, Mohamed Ali AA, Kholef EFM, Elsewify WAE. Golgi Protein 73 versus Alpha-Fetoprotein as a New Biomarker in Early Diagnosis of Hepatocellular Carcinoma. Int J Gen Med. 2020; 13:193-200.

https://doi.org/10.2147/IJGM.S253622

PMID: $\underline{32547160}$

4. Huang $X$, Qin F, Meng $Q$, Dong $M$. Protein tyrosine phosphatase receptor type D (PTPRD)-mediated signaling pathways for the potential treatment of hepatocellular carcinoma: a narrative review. Ann Transl Med. 2020; 8:1192.

https://doi.org/10.21037/atm-20-4733

PMID:33241041

5. Elsewify WAE, Hassan EA, Mekky MA, Abd El-Rehim ASE, Sayed ZEA, Abdel Malek MO, ElMelegy TTH, Sabry A. Usefulness of Circulating Methylated p16 as a Noninvasive Molecular Biomarker for Hepatitis CRelated Hepatocellular Carcinoma with Normal Serum Alpha-Fetoprotein Levels. Int J Gen Med. 2020; 13:147-55. https://doi.org/10.2147/IJGM.S249272 PMID:32431531

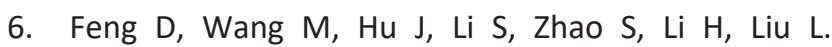
Prognostic value of the albumin-bilirubin grade in patients with hepatocellular carcinoma and other liver diseases. Ann Transl Med. 2020; 8:553. https://doi.org/10.21037/atm.2020.02.116 PMID: $\underline{32411776}$ 
7. Ren $X$, Wang $X$, Peng $B$, Liang $Q$, Cai $Y$, Gao K, Hu Y, Xu $Z$, Yan Y. Significance of TEAD Family in Diagnosis, Prognosis and Immune Response for Ovarian Serous Carcinoma. Int J Gen Med. 2021; 14:7133-43.

https://doi.org/10.2147/IJGM.S336602 PMID: $\underline{34737608}$

8. Sturbaut $M$, Bailly F, Coevoet $M$, Sileo $P$, Pugniere $M$, Liberelle M, Magnez R, Thuru X, Chartier-Harlin MC, Melnyk P, Gelin M, Allemand F, Guichou JF, Cotelle P. Discovery of a cryptic site at the interface 2 of TEAD - Towards a new family of YAP/TAZ-TEAD inhibitors. Eur J Med Chem. 2021; 226:113835.

https://doi.org/10.1016/i.ejmech.2021.113835 PMID:34509860

9. Yuan Y, Salinas Parra N, Chen Q, Iglesias-Bartolome R. Oncogenic Hedgehog-Smoothened Signaling Depends on YAP1-TAZ/TEAD Transcription to Restrain Differentiation in Basal Cell Carcinoma. J Invest Dermatol. 2022; 142:65-76.e7.

https://doi.org/10.1016/i.jid.2021.06.020

PMID:34293352

10. Yang $\mathrm{W}$, Yang $\mathrm{S}$, Zhang $\mathrm{F}$, Cheng $\mathrm{F}$, Wang $\mathrm{X}$, Rao J. Influence of the Hippo-YAP signalling pathway on tumor associated macrophages (TAMs) and its implications on cancer immunosuppressive microenvironment. Ann Transl Med. 2020; 8:399.

https://doi.org/10.21037/atm.2020.02.11 PMID:32355843

11. Liu Y, Zhang $\mathrm{Q}$, Wu J, Zhang H, Li X, Zheng Z, Luo M, Li L, Xiang Y, Yang F, Wu L. Long Non-Coding RNA A2MAS1 Promotes Breast Cancer Progression by Sponging microRNA-146b to Upregulate MUC19. Int J Gen Med. 2020; 13:1305-16.

https://doi.org/10.2147/IJGM.S278564

PMID:33273850

12. Pearson JD, Huang K, Pacal M, McCurdy SR, Lu S, Aubry A, Yu T, Wadosky KM, Zhang L, Wang T, Gregorieff A, Ahmad M, Dimaras $H$, et al. Binary pancancer classes with distinct vulnerabilities defined by pro- or anti-cancer YAP/TEAD activity. Cancer Cell. 2021; 39:1115-34.e12.

https://doi.org/10.1016/i.ccell.2021.06.016 PMID:34270926

13. Tocci P, Cianfrocca R, Sestito R, Rosanò L, Di Castro V, Blandino G, Bagnato A. Endothelin-1 axis fosters YAPinduced chemotherapy escape in ovarian cancer. Cancer Lett. 2020; 492:84-95.

https://doi.org/10.1016/j.canlet.2020.08.026 PMID: $\underline{32860850}$

14. Xiu Z, Liu J, Wu X, Li X, Li S, Wu X, Lv X, Ye H, Tang X. Cytochalasin $H$ isolated from mangrove-derived endophytic fungus inhibits epithelial-mesenchymal transition and cancer stemness via YAP/TAZ signaling pathway in non-small cell lung cancer cells. J Cancer. 2021; 12:1169-78.

https://doi.org/10.7150/ica.50512

PMID: 33442415

15. Gao R, Kalathur RKR, Coto-Llerena M, Ercan C, Buechel D, Shuang S, Piscuoglio S, Dill MT, Camargo FD, Christofori G, Tang F. YAP/TAZ and ATF4 drive resistance to Sorafenib in hepatocellular carcinoma by preventing ferroptosis. EMBO Mol Med. 2021; 13:e14351.

https://doi.org/10.15252/emmm.202114351 PMID: 34664408

16. Tang R, Hua J, Xu J, Liang C, Meng Q, Liu J, Zhang B, Yu $X$, Shi $S$. The role of ferroptosis regulators in the prognosis, immune activity and gemcitabine resistance of pancreatic cancer. Ann Transl Med. 2020; 8:1347.

https://doi.org/10.21037/atm-20-2554a

PMID:33313092

17. Liu Y, Zhang X, Zhang J, Tan J, Li J, Song Z. Development and Validation of a Combined Ferroptosis and Immune Prognostic Classifier for Hepatocellular Carcinoma. Front Cell Dev Biol. 2020; 8:596679.

https://doi.org/10.3389/fcell.2020.596679

PMID:33425905

18. Yan $Y$, Liang $Q$, Xu Z, Huang J, Chen X, Cai Y, Peng B, Yi Q. Downregulated Ferroptosis-Related Gene STEAP3 as a Novel Diagnostic and Prognostic Target for Hepatocellular Carcinoma and Its Roles in Immune Regulation. Front Cell Dev Biol. 2021; 9:743046.

https://doi.org/10.3389/fcell.2021.743046

PMID:34790664

19. Sun T, Chi JT. Regulation of ferroptosis in cancer cells by YAP/TAZ and Hippo pathways: The therapeutic implications. Genes Dis. 2020; 8:241-9.

https://doi.org/10.1016/j.gendis.2020.05.004 PMID:33997171

20. Yang $W H$, Ding $C C$, Sun $T$, Rupprecht $G$, Lin $C C$, Hsu D, Chi JT. The Hippo Pathway Effector TAZ Regulates Ferroptosis in Renal Cell Carcinoma. Cell Rep. 2019; 28:2501-8.e4.

https://doi.org/10.1016/j.celrep.2019.07.107 PMID: $\underline{1484063}$

21. Galani V, Varouktsi A, Papadatos SS, Mitselou A, Sainis I, Constantopoulos S, Dalavanga Y. The role of apoptosis defects in malignant mesothelioma pathogenesis with an impact on prognosis and treatment. Cancer Chemother Pharmacol. 2019; 84:241-53.

https://doi.org/10.1007/s00280-019-03878-3

PMID: $\underline{1119375}$ 
22. Tang Z, Li C, Kang B, Gao G, Li C, Zhang Z. GEPIA: a web server for cancer and normal gene expression profiling and interactive analyses. Nucleic Acids Res. 2017; 45:W98-102.

https://doi.org/10.1093/nar/gkx247 PMID:28407145

23. Chandrashekar DS, Bashel B, Balasubramanya SAH, Creighton CJ, Ponce-Rodriguez I, Chakravarthi BVS, Varambally S. UALCAN: A Portal for Facilitating Tumor Subgroup Gene Expression and Survival Analyses. Neoplasia. 2017; 19:649-58.

https://doi.org/10.1016/j.neo.2017.05.002 PMID:28732212

24. Tang Z, Li C, Zhang K, Yang M, Hu X. GE-mini: a mobile APP for large-scale gene expression visualization. Bioinformatics. 2017; 33:941-3. https://doi.org/10.1093/bioinformatics/btw775 PMID:28065896

25. Rhodes DR, Kalyana-Sundaram S, Mahavisno V, Varambally R, Yu J, Briggs BB, Barrette TR, Anstet MJ, Kincead-Beal C, Kulkarni P, Varambally S, Ghosh D, Chinnaiyan AM. Oncomine 3.0: genes, pathways, and networks in a collection of 18,000 cancer gene expression profiles. Neoplasia. 2007; 9:166-80.

https://doi.org/10.1593/neo.07112

PMID:17356713

26. Yuan Q, Sun N, Zheng J, Wang Y, Yan X, Mai W, Liao Y, Chen X. Prognostic and Immunological Role of FUN14 Domain Containing 1 in Pan-Cancer: Friend or Foe? Front Oncol. 2020; 9:1502. https://doi.org/10.3389/fonc.2019.01502 PMID:31998650

27. Gao J, Aksoy BA, Dogrusoz U, Dresdner G, Gross B, Sumer SO, Sun $Y$, Jacobsen A, Sinha R, Larsson E, Cerami E, Sander C, Schultz N. Integrative analysis of complex cancer genomics and clinical profiles using the cBioPortal. Sci Signal. 2013; 6:pl1. https://doi.org/10.1126/scisignal.2004088 PMID:23550210

28. Zhao C, Sahni S. String correction using the DamerauLevenshtein distance. BMC Bioinformatics. 2019 (Suppl 11); 20:277. https://doi.org/10.1186/s12859-019-2819-0 PMID:31167641

29. Liao Y, Wang J, Jaehnig EJ, Shi Z, Zhang B. WebGestalt 2019: gene set analysis toolkit with revamped Uls and APIs. Nucleic Acids Res. 2019; 47:W199-205. https://doi.org/10.1093/nar/gkz401 PMID:31114916

30. Kanehisa M, Furumichi $M$, Tanabe $M$, Sato $Y$, Morishima K. KEGG: new perspectives on genomes, pathways, diseases and drugs. Nucleic Acids Res. 2017; 45:D353-61. https://doi.org/10.1093/nar/gkw1092 PMID:27899662

31. Li T, Fu J, Zeng Z, Cohen D, Li J, Chen Q, Li B, Liu XS. TIMER2.0 for analysis of tumor-infiltrating immune cells. Nucleic Acids Res. 2020; 48:W509-14.

https://doi.org/10.1093/nar/gkaa407

PMID: $\underline{32442275}$

32. Liang JY, Wang DS, Lin HC, Chen XX, Yang $H$, Zheng $Y$, $\mathrm{Li}$ YH. A Novel Ferroptosis-related Gene Signature for Overall Survival Prediction in Patients with Hepatocellular Carcinoma. Int J Biol Sci. 2020; 16:2430-41.

https://doi.org/10.7150/ijbs.45050

PMID: $\underline{32760210}$

33. Xu Z, Peng B, Liang Q, Chen X, Cai Y, Zeng S, Gao K, Wang X, Yi Q, Gong Z, Yan Y. Construction of a Ferroptosis-Related Nine-IncRNA Signature for Predicting Prognosis and Immune Response in Hepatocellular Carcinoma. Front Immunol. 2021; 12:719175.

https://doi.org/10.3389/fimmu.2021.719175 PMID: $\underline{34603293}$

34. Yao Y, Chen Z, Zhang H, Chen C, Zeng M, Yunis J, Wei Y, Wan Y, Wang N, Zhou M, Qiu C, Zeng Q, Ong HS, et al. Selenium-GPX4 axis protects follicular helper T cells from ferroptosis. Nat Immunol. 2021; 22:1127-39.

https://doi.org/10.1038/s41590-021-00996-0 PMID:34413521

35. Wei R, Qiu H, Xu J, Mo J, Liu Y, Gui Y, Huang G, Zhang $\mathrm{S}$, Yao H, Huang X, Gan Z. Expression and prognostic potential of GPX1 in human cancers based on data mining. Ann Transl Med. 2020; 8:124.

https://doi.org/10.21037/atm.2020.02.36

PMID:32175417

36. Du J, Wan Z, Wang C, Lu F, Wei M, Wang D, Hao Q. Designer exosomes for targeted and efficient ferroptosis induction in cancer via chemophotodynamic therapy. Theranostics. 2021; 11:8185-96.

https://doi.org/10.7150/thno.59121

PMID: $\underline{34373736}$

37. Wang Q, Bin C, Xue Q, Gao Q, Huang A, Wang K, Tang N. GSTZ1 sensitizes hepatocellular carcinoma cells to sorafenib-induced ferroptosis via inhibition of NRF2/GPX4 axis. Cell Death Dis. 2021; 12:426. https://doi.org/10.1038/s41419-021-03718-4 PMID: $\underline{3931597}$

38. Zinatizadeh MR, Miri SR, Zarandi PK, Chalbatani GM, Rapôso C, Mirzaei HR, Akbari ME, Mahmoodzadeh H. The Hippo Tumor Suppressor Pathway (YAP/TAZ/TEAD/MST/LATS) and EGFR-RAS-RAF-MEK in cancer metastasis. Genes Dis. 2019; 8:48-60. 
https://doi.org/10.1016/j.gendis.2019.11.003

PMID: $\underline{3569513}$

39. Huh HD, Kim DH, Jeong HS, Park HW. Regulation of TEAD Transcription Factors in Cancer Biology. Cells. 2019; 8:600.

https://doi.org/10.3390/cells8060600

PMID:31212916

40. Xie W, Xiao W, Tang K, Zhang L, Li Y. Yes-Associated Protein 1: Role and Treatment Prospects in Orthopedic Degenerative Diseases. Front Cell Dev Biol. 2020; 8:573455.

https://doi.org/10.3389/fcell.2020.573455

PMID: $\underline{3178690}$

41. Zhou A, Yu H, Liu J, Zheng J, Jia Y, Wu B, Xiang L. Role of Hippo-YAP Signaling in Osseointegration by Regulating Osteogenesis, Angiogenesis, and Osteoimmunology. Front Cell Dev Biol. 2020; 8:780.

https://doi.org/10.3389/fcell.2020.00780 PMID:32974339

42. Xu W, Zhang $M$, Li Y, Wang $Y$, Wang K, Chen Q, Zhang $\mathrm{R}$, Song $\mathrm{W}$, Huang $\mathrm{Q}$, Zhao $\mathrm{W}$, Wu J. YAP manipulates proliferation via PTEN/AKT/mTOR-mediated autophagy in lung adenocarcinomas. Cancer Cell Int. 2021; 21:30.

https://doi.org/10.1186/s12935-020-01688-9

PMID:33413409

43. Lin CC, Yang WH, Lin YT, Tang $X$, Chen PH, Ding CC, Qu DC, Alvarez JV, Chi JT. DDR2 upregulation confers ferroptosis susceptibility of recurrent breast tumors through the Hippo pathway. Oncogene. 2021; 40:2018-34. https://doi.org/10.1038/s41388-021-01676-x

PMID: $\underline{3603168}$

44. Wu J, Minikes AM, Gao M, Bian H, Li Y, Stockwell BR, Chen $Z N$, Jiang $X$. Intercellular interaction dictates cancer cell ferroptosis via NF2-YAP signalling. Nature. 2019; 572:402-6. https://doi.org/10.1038/s41586-019-1426-6 PMID:31341276

45. Zhu G, Murshed A, Li H, Ma J, Zhen N, Ding M, Zhu J, Mao S, Tang X, Liu L, Sun F, Jin L, Pan Q. OGlcNAcylation enhances sensitivity to RSL3-induced ferroptosis via the YAP/TFRC pathway in liver cancer. Cell Death Discov. 2021; 7:83. https://doi.org/10.1038/s41420-021-00468-2 PMID:33863873

46. Brewer CM, Nelson BR, Wakenight $P$, Collins SJ, Okamura DM, Dong XR, Mahoney WM Jr, McKenna A, Shendure J, Timms A, Millen KJ, Majesky MW. Adaptations in Hippo-Yap signaling and myofibroblast fate underlie scar-free ear appendage wound healing in spiny mice. Dev Cell. 2021; 56:2722-40.e6. https://doi.org/10.1016/j.devcel.2021.09.008 PMID: $\underline{34610329}$ 


\section{SUPPLEMENTARY MATERIALS}

\section{Supplementary Tables}

Supplementary Table 1. The databases used in the analysis of the roles of TEAD family.

\begin{tabular}{|c|c|c|}
\hline Databases & URL & Refs \\
\hline GEPIA & http://gepia.cancer-pku.cn/ & {$[22]$} \\
\hline UALCAN & http://ualcan.path.uab.edu/analysis.html & {$[23]$} \\
\hline GE-mini & http://gemini.cancer-pku.cn/ & [24] \\
\hline Oncomine3.0 & https://www.oncomine.org/resource/login.html & {$[25]$} \\
\hline Kaplan-Meier plotter & $\underline{\mathrm{http}}: / / \mathrm{kmplot} . \mathrm{com} /$ analysis/ & [26] \\
\hline cBioPortal & http://www.cbioportal.org/ & [27] \\
\hline STRING & https://string-db.org/cgi/input.pl & [28] \\
\hline WebGestalt & http://www.webgestalt.org/ & [29] \\
\hline KEGG & https://www.genome.jp/kegg/ & [30] \\
\hline TIMER2.0 & https://cistrome.shinyapps.io/timer/ & [31] \\
\hline
\end{tabular}

Please browse Full Text version to see the data of Supplementary Table 2.

Supplementary Table 2. The coexpression genes of TEAD family-associated in HCC patients. 